\title{
Molecular epidemiology of VIM and IMP-producing Klebsiella isolates from urinary tract infection patients in an educational hospital in Shahrekord, Iran
}

Farshad Kakian ${ }^{1}$, Mohamad Hoseein Rezaei ${ }^{2}$, Masoud Amiri ${ }^{3}$, Saeid Janabadi ${ }^{1}$, Behnam Zamanzad $^{4, *}$, Abolfazl Gholipour ${ }^{4}$, Kourosh Naderi ${ }^{2}$

${ }^{1}$ Department of Bacteriology and Virology, School of Medicine, Shiraz University of Medical Sciences, Shiraz, Iran

2 Cellular and Molecular Research Center, Shahrekord University of Medical Sciences, Shahrekord, Iran

${ }^{3}$ Department of Epidemiology, Erasmus Medical Center, Rotterdam, The Netherlands

${ }^{4}$ Department of Microbiology and Immunology, School of Medicine, Shahrekord University of Medical Sciences, Shahrekord, Iran.

*. Corresponding author: Behnam Zamanzad, Department of Microbiology and Immunology, Cellular and Molecular Research Center, Shahrekord University of Medical Sciences, Shahrekord, IR Iran, Tel: +983833346732, Fax: +98383334911, E-mail: zamanzad@ skums.ac.ir.

Cite this article: Kakian, F.; Rezaei, M.H.; Amiri, M.; Janabadi, S.; Zamanzad, B.; Gholipour, A.; Naderi, K. Molecular epidemiology of VIM and IMP-producing Klebsiella isolates from urinary tract infection patients in an educational hospital. Int J Epidemiol Health Sci 2020;1(2): e02. Doi:10.51757/IJEHS.1.2.2020.44700.

\begin{abstract}
Background and aim: Klebsiella is an opportunistic organism that is the cause of many nosocomial infections. The present study was designed to investigate the molecular epidemiology of Verona integron-encoded metallo- $\beta$ lactamase (VIM) and Imipenemase (IMP)-producing Klebsiella isolates in patients with urinary tract infection (UTI) in an educational hospital in Shahrekord, Iran.

Methods: In a cross-sectional study, from 234 urine samples, 80 isolates of Klebsiella were identified with biochemical tests. In order to determine the production of Metallo- $\beta$-lactamases (MBLs), Modified Hodge Test (MHT), EDTA Disc Synergy (EDS) test and Ampicilin C (AmpC) disc test were performed. The frequency of VIM and IMP genes was determined after DNA-amplification with PCR by electrophoresis technique. As an internal control in PCTR, 16SrRNA was considered.

Results: Phenotypic tests showed that out of the 80 isolates, $18(22.5 \%), 18(22.5 \%)$ and $10(12.5 \%)$ isolates were positive for MHT, EDS and AmpC disc test, respectively. Following DNA amplification by PCR, the genes of interest were analyzed by electrophoresis technique. The findings were as follows: 22 isolates (27.5\%) carried the VIM gene, but the IMP gene was not found in any of the isolates.

Conclusions: Expansion of Klebsiella strains that produce MBLs is a severe threat to health centers and public health. The findings of this study showed that Klebsiella may produce MBLs. These enzymes can in turn degrade carbapenem antibiotics, which are considered as a last resort in the treatment of multidrug-resistant (MDR) infection.
\end{abstract}

Keywords: Klebsiella, Antibiotic resistance, Verona integron-encoded metallo- $\beta$-lactamase (VIM), Imipenemase (IMP), Iran 


\section{Introduction}

Urinary tract infection (UTI), affecting 150 million people yearly, are an important threat to human health. Between 30 to 40 percent of UTI are due to nosocomial infections $(1,2)$. Klebsiella pneumoniae and Escherichia coli are known to cause more hospital infections in comparison to other bacteria of the Enterobacteriaceae family (3). Klebsiella is an opportunistic organism, which may lead to the development of severe diseases such as pneumonia, septicemia and UTI (4). Klebsiella can be present in hospitals, persist on environmental surfaces and colonize diverse parts of the human body. Therefore, the patients are at high risk of being infected by Klebsiella during their hospital stay. The frequent use of microbial agents and thereby the continuous exposure of this bacterium to many antibiotics has contributed to the spread of the multidrug-resistant strains, which has made it challenging for healthcare settings to control infections with this bacterium $(5,6)$. This bacterium is a dangerous pathogen affecting humans and acts as the primary source for hospitalrelated infections. Due to the limited treatment options, infections with this bacterium are associated with high morbidity and mortality (7). Similar to other countries in the world, antibiotic resistance and nosocomial infections are also major problems in Iran (8).

Antibiotic resistance has made treating UTI more challenging. There are more than 12,000 deaths per year from UTI in USA (9), with a prevalence of $4.92 \%$ among Iranian children (10) and $14.96 \%$ in Northern Iran (11). The carbapenems consist of Ertapenem, Imipenem, Doripenem and Meropenem. The most essential resistant isolates of Klebsiella pneumoniae are carbapenem and cephalosporin-resistant strains (12-14). The presence of MBLs is responsible for resistance against carbapenem antibiotics. Resistance caused by MBLs often depends on the plasmid (15, 16). Carbapenemases belong to the molecular classes A, B and D, according to the Ambler classification. MBL's belong to class B. Based on their molecular structure, MBLs are divided into six categories: VIM, IMP, GIM, SIM, SPM, and AIM. The VIM, IMP and SMP-1 genes were identified in Pseudomonas aeruginosa, Acinetobacter baumannii and other members of the Enterobacteriaceae family such as Klebsiella (17-20). Molecular epidemiological studies are important to identify the source of infections so that the healthcare settings come up with innovative, preventative strategies to control the transmission of bacteria between patients and even among wards. This study aimed to explore the epidemiology of VIM and IMP genes in UTI patients in Shahrekord city, in the center of Iran.

\section{Materials and Methods}

\section{Bacterial isolation}

In this cross-sectional study, among the 234 collected urine samples, 80 isolates of Klebsiella were identified in several sections of an educational hospital in Shahrekord, in Iran. From May to October 2016, the patients of different wards (urology, gynaecology, internal medicine and ICU) who were infected by UTI within 48-72 hours after hospitalization and then urine culture was done for them and their culture was negative. The mean age of the participants was 38.6 years. The minor and major prevalence of isolated bacteria was allocated to the urology and ICU ward, respectively. To collect urine, the outer part of the urethra was firstly washed, after which the urine was collected. After the collection of urine, the samples were cultured on blood agar and EMB mediums (Himedia). Following the growth of bacteria on the 
mediums then different biochemical identifier tests such as Triple Sugar Iron test (TSI), Simon citrate, Methyl Red / Voges-Proskauer (MR/VP), SIM (Sulfide, Indole, Motility), Oxidase and Catalase were performed on the isolates.

\section{Phenotypic detection of MBLs enzymes}

Phenotypic tests, including Modified Hodge Test (MHT), EDTA Disc Synergy (EDS) test and AmpC disc test were performed to detect the MBLs produced by the isolates.

\section{Modified Hodge Test}

In the running MHT, E. coli (ATCC 25922) was considered as a negative control and Klebsiella pneumonia (ATCC 1705) was considered as a positive control. For this test, a bacterial suspension equivalent to $0.5 \mathrm{McFarland}$ standard was prepared from fresh cultures of E. coli (ATCC 25922) and diluted in 1:10 by sterile distilled water, and then passaged on Mueller-Hinton agar. Besides, a meropenem disc (10 $\mu \mathrm{g}$ ) was placed in the center of the plate and passaging of the colony of the unknown samples from the edge of the disc to the edge of the plate linearly in four directions. After having taken these actions, the plates were maintained at $37^{\mathrm{C} \circ}$ overnight. Then, appeared clover leaf shape in the inhibition site of bacterial growth resulted from the production of MBL by the tested isolates $(21,22)$.

\section{EDTA Disc Synergy test (EDS)}

In this test, a 0.5 M EDTA solution and a meropenem disc $(10 \mu \mathrm{g})$ were used. In order to perform this test, suspension samples equivalent to $0.5 \mathrm{McF}$ arland were passaged on Mueller-Hinton agar. A meropenem disc $(10 \mu \mathrm{g})$ was placed on the plate. The blank disc (containing $10 \mathrm{ml}$ of a $0.5 \mathrm{M}$ EDTA solution) was placed at a $10 \mathrm{~mm}$ distance from the meropenem disc. The plate was incubated overnight at $37^{\mathrm{Co}}$. Increased growth inhibition zone between two discs is an indicator of MBL production (22).

\section{AmpC disc test}

The AmpC disc test method was used to identify AmpC $\beta$-lactamase. In this setting, $0.5 \mathrm{M}$ of a McFarland suspension of E. coli ATCC 25922 was passaged on Mueller-Hinton culture medium. Besides, a cefoxitin disc $(30 \mu \mathrm{g})$ was placed on the plate near the blank disc, which was exposed to normal saline. Then the intended colony were added to the blank disc. To be able to assess whether there was AmpC $\beta$ lactamase production, the growth area of the cefoxitin disc in the vicinity of the blank disc was observed (23, 24). The availability of a dentin in the growth are of the cefoxitin disc was an indication of AmpC $\beta$ lactamase production.

\section{Detection of VIM and IMP genes}

In order to perform PCR, DNA was extracted by the conventional boiling method. In this method, $1 \mathrm{ml}$ of sterile distilled water was poured in a $1.5 \mathrm{ml}$ microtube. Then 4 to 5 fresh bacterial colonies were dissolved, after which the microtubes were placed on a heated plate at $95^{\mathrm{C} \circ}$ for 15 minutes to boil. The samples were centrifuged at $14000 \mathrm{rpm}$ for 5 minutes. The transparent liquid containing bacterial DNA was removed for PCR. In this study, Acinetobacter bumanni AC54/97 was the positive control for the IMP gene and Pseudomonas aeruginosa PO510 for the VIM gene $(6,25)$. Furthermore, 16srRNA was considered as an internal control (26). The primers used in this study for the VIM and the IMP genes, were 
designed earlier by the Oligo 7 software in the study of Rezvan Vafapour in the Cellular and Molecular Research Center in Shahrekord, in Iran (approval of Vafapour study code: 1392-01-74-1917). The electrophoresis technique was performed on the polyacrylamide gel after completing the PCR steps.
The polyacrylamide gel was stained by the silver nitrate method (Figure 2). All the specimens contained 16SrRNA as internal control genes, which confirmed the presence of this genotype gene for Klebsiella.

Table 1. Primers used for the VIM and the IMP by PCR

\begin{tabular}{lc}
\hline Primer Sequence (5' to $\left.3^{\prime}\right)$ & Size (bp) \\
\hline VIM F TGGTTGTATACGTCCCGTCA & 206 \\
VIM R TGTGTGCTGGAGCAAGTCTA & \\
\hline IMP F TAACGGGTGGGGCGTTGTTCCT & 179 \\
IMP R CGCCCGTGCTGTCGCTATGAAA & 420 \\
\hline 16Sr RNA F AGGCCTTCGGGTTGTAAAGT & \\
16Sr RNA R ACCTCCAAGTCGACATCGTT &
\end{tabular}

VIM: Verona integron-encoded metallo- $\beta$-lactamase; IMP: Imipenemase; PCR: Polymerase chain reaction.

\section{Statistical analysis}

Descriptive statistics (i.e. frequency, percentage, and the mean and standard deviation) and inferential statistics (Fisher's exact test) were performed in order

\section{Results}

\section{Resistance to Meropenem and Imipenem}

After incubating the plates containing the meropenem and imipenem discs, the diameters of the inhibition zones were measured and interpreted by following the guidelines of the Clinical Laboratory Standards Institute (CLSI). In the disc diffusion method, 24 isolates were resistant, and 10 isolates had a moderate sensitivity for meropenem and imipenem. The results of phenotypic tests for detecting MBLs Strains that were resistant to Meropenem and Imipenem based on the results obtained by the E-Test, to analyze the data. Hereby, $\mathrm{P}<0.05$ was considered as $\alpha$.

were used in Phenotypic tests to detect MBL enzymes. The results were as following: 18 isolates $(22.5 \%)$ were MHT positive. Likewise, in the EDS test, 18 isolates (22.5\%) were positive. 
The presence of a notch in the side of the disc reflects the production of AmpC $\beta$-lactamase (Figure 1). In this test, $10(12.5 \%)$ isolates had an $\beta$-lactamase.
Results of the phenotypic tests, which were used to detect MBL enzymes, are presented in Table 2.

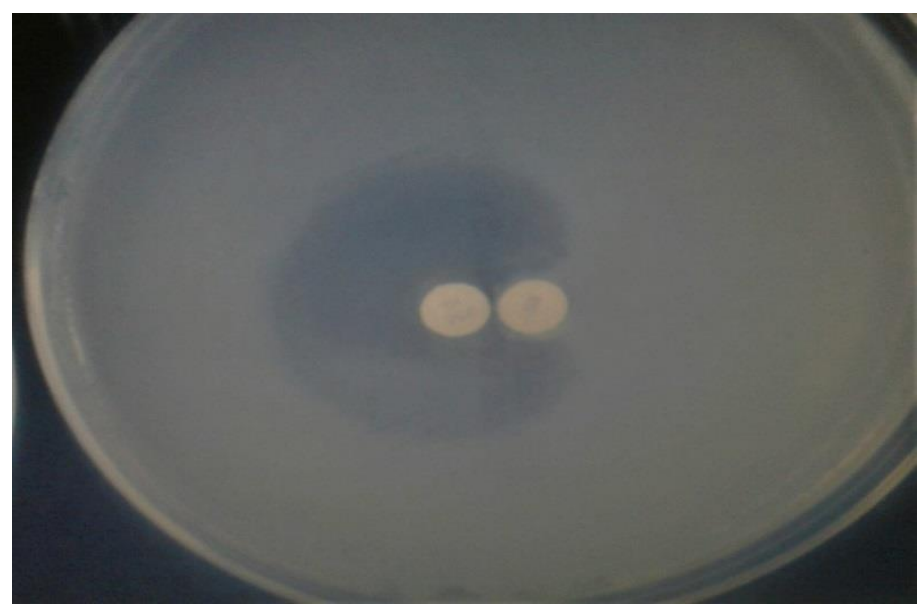

Figure 1. AmpC Disc Test. The notches on the side of the disc represent AmpC B-lactamase producing Klebsiella, which prevents the creation of a complete halation around the cefoxitin disk by E. coli ATCC 25922.

Table 2. Results of the phenotypic tests for detecting MBL enzymes

\begin{tabular}{llllll}
\hline Samples & Disc-diffusion & E-Test & MHT & EDS Test & AmpC Disc Test \\
\hline $\mathrm{N}=80$ & 24 & 24 & 18 & 18 & 10 \\
& $(30 \%)$ & $(30 \%)$ & $(22.5 \%)$ & $(22.5 \%)$ & $(12.5 \%)$ \\
\hline
\end{tabular}

E-Test: Epsilon Test; MHT: Modified Hodge Test; EDS: EDTA Disc Synergy; AmpC (Ampicillin C)

\section{The results of genotypic tests}

Gene detection by PCR and electrophoresis technique showed that 22 isolates (27.5\%) carried the VIM gene, but the IMP gene was not observed in any of the isolates. 


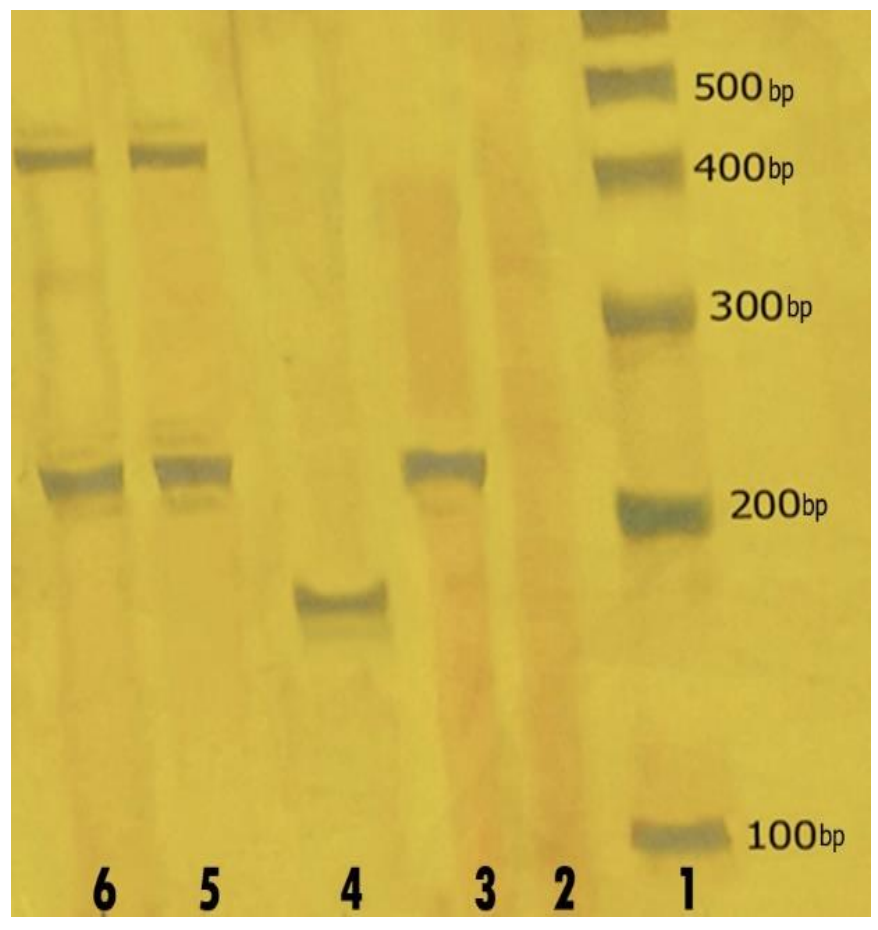

Figure 2. Colored gel of polyacrylamide. Column 1: Ladder (bp). Column 2: Negative control. Column 3: Positive control of the VIM gene (P.aeruginosa PO510). Column 4: Positive control of the IMP gene (A.baumanii AC54 / 97). Columns 5 and 6: The VIM gene and an internal control gene 16srRNA without the IMP.

\section{Discussion}

Our findings showed that 24 isolates were resistant to Meropenem and Imipenem. In addition, PCR showed that 22 isolates, carried the VIM gene, but the IMP gene was not found in any isolate.

Nowadays, bacterial resistance against several antibiotics has become a global concern. Genes encoding for MBL's are found more frequently on integrins, particularly class 1 integrins, which has the genetic mobility of these elements, promoting the release of enzymes in different parts of health care centers (27). As with studies in Columbia, Japan, and Italy, the VIM and the IMP were observed without the use of Carbapenem antibiotics, confirming the transfer of these genes among different species of bacteria (27, 28).

In a study conducted by Nobari and colleagues in 2014, among 180 isolates of Klebsiella, 42 isolates were resistant to Meropenem, 29 isolates were resistant to Ertapenem and 14 isolates were resistant to Imipenem (29). In a another similar study conducted by Sahin and colleagues in 2015, from 43 isolates of E. coli and Klebsiella, 35 isolates were carbapenemase-resistant and produced MBL enzymes (30). The Center for Disease Control (CDC) has provided MHT testing to detect MBLs (31). In this test, the results are more reliable when used with the 
Imipenem disc with 0.5 molar EDTA or $50 \mathrm{mM}$ ZNSO4 (32). The results of previous studies showed that MHT has less favorable outcomes than other tests, ranging from 14.8 to $51.16 \%$ (32-34), consistent with our results. The present study also revealed that from the 80 isolates, 18,18 and 10 isolates were positive for MHT, EDS and AmpC, respectively.

In another study conducted by Kumar and colleagues in 2015, from 28 isolates of E. coli, six isolates were MHT test positive (35). Also, in the study of Sahin and colleagues, from 43 isolates of Klebsiella and E. coli, 35 isolates were MHT test positive, but the VIM and the IMP genes not found by PCR, similar to our findings with no detection of the IMP gene (30). In the study of Kanchanadevi and colleagues in 2016, from 76 isolates, 12 isolates were EDS test positive, which is consistent with our results (36). Also, in a similar study conducted in India, the prevalence of AmpC in Klebsiella was $6.18 \%(36,37)$. In another study of Kanchanadevi and colleagues, from 105 isolates, 2\% were positive for AmpC $\beta$-lactamase (38).

In the study of Mousavian and colleagues in 2016, the molecular prevalence of the IMP gene in Klebsiella isolates, was zero (39). Also, in the Tawfik and colleagues' study in 2010, from 15 MBL enzyme positive isolates, all the isolates were the VIM positive, but the IMP gene was not found, similar to our results (40). Zayghami and colleagues reported that among 100 isolates of Klebsiella and E. coli, the prevalence of the VIM and the IMP genes in Klebsiella was $41 \%$, but these genes not found in E. coli, that shows the difference between the outbreak of these genes among different bacteria (41).

In the present study, the prevalence of carbapenemresistant enzymes was high and significant. Arbitrary use of antibiotics, high prescription antibiotics by doctors, shortage specialist physician, experimental treatment of doctors, as well as the lack of sufficient knowledge of people about how to use the drug, they can be one of the reasons. Also, positive phenotypic tests may be due to the presence of other MBLs genes such as NDM, GIM, SIM, SPM. Therefore, it is suggested that the presence of these genes be investigated in future studies.

\section{Conclusion}

Klebsiella, with resistance to Meropenem and Imipenem, is considered as a substantial public health challenge to patients. This need to direct more attention to antimicrobial resistance by monitoring and surveillance. Furthermore, it would be essential that antibiotics be prescribed appropriately to prevent the emergence and spread of bacterial strains with resistance to carbapenemase in hospitals and the community.

\section{Conflict of Interests}

None.

\section{Ethical Statement}

The study protocol was obtained by the ethics code of IR. SKUMS. REC.1394.282 from Shahrekord University of Medical Sciences, Shahrekord, Iran.

\section{Acknowledgments}

The authors are thankful to the staffs of the Students Research Committee, Shahrekord University of Medical Sciences, Shahrekord, Iran.

\section{Funding/Support}

This research was supported by the budget of research projects of the Shahrekord University of Medical Sciences (Number of project: 2057)

\section{References}

1. Karchmer, T.B., Giannetta, E.T., Muto, C.A., Strain, B.A. and Farr, B.M.. A randomized 
crossover study of silver-coated urinary catheters in hospitalized patients. Arch Int Med 2000;160(21):3294-8.

2. Waller, T.A.,Pantin, S.A.L., Yenior, A.L. and Pujalte, G.G.A.Urinary tract infection antibiotic resistance in the United States. Prim Care 2018; 45(3): 455-466.

3. Baroud, M., Dandache, I., Araj, G.F., Wakim, R., Kanj, S., Kanafani, Z., Kheirallah, M., Sabra, A., Shehab, M., Dbaibo, G. and Matar, G.M. Underlying mechanisms of carbapenem resistance in extendedspectrum $\quad \beta$-lactamase-producing Klebsiella pneumoniae and Escherichia coli isolates at a tertiary care centre in Lebanon: role of OXA-48 and NDM-1 carbapenemases. Int $\mathrm{J}$ Antimicrob Agents 2013;41(1):75-9.

4. Essack, S.Y. Treatment options for extendedspectrum $\beta$-lactamase-producers. FEMS Microbiol letters 2000;190(2):181-4.

5. Heidary, M., Bahramian, A., Goudarzi, H., Eslami, G., Hashemi, A and Khoshnood,S. To study the association between AcrAB and Qep A efflux pumps and ciprof-loxacin resistance among Escherichia coli and Klebsiella pneumoniae clinical strains. Arak Med Univ J 2016;19(4):1-10.

6. Kakian, F., Shahini Shams Abadi, M., Zamanzad, B., Najafi, H., Amiri, M. and Gholipour, A..Frequency assessment of OXA-10 and PER $\beta$-lactamase genes and determination of minimum inhibitory concentration in klebsiella strains isolated from urinary tract infections. Jundishapur J Microbiol 2019; 12(2):e65500.

7. Pogue, J. M.,Kaye, K.S., Cohen, D.A. and Marchaim, D. Appropriate antimicrobial therapy in the era of multidrug-resistant human pathogens. Clin Microbiol Infect 2015;21(4): 302-312.
8. Behzadnia, S., Davoudi, A., Rezaei, M.S. and Ahangarkani, F. Nosocomial infections in pediatric population and antibiotic resistance of the causative organisms in north of Iran. Iran Red Crescent Med J 2014; 16(2):e14562.

9. Kumar, M. S. and Das, A.P. Emerging nanotechnology based strategies for diagnosis and therapeutics of urinary tract infections: a review. Adv colloid interface sci 2017; 249:53-65.

10. Bouya, S., Koochakzai, M., Rafiemanesh, H., Balouchi, A. and Hesaraki, M.. The Prevalence of Urinary Tract Infections in Iranian Children: A MetaAnalysis and Systematic Review. J Clin Diagnos Res. 2018; 12(8): SE01-SE04.

11. Mihankhah A, Khoshbakht R, Raeisi M, Raeisi V. Prevalence and antibiotic resistance pattern of bacteria isolated from urinary tract infections in Northern Iran. J Res Med Sci 2017;22:108.

12. Meletis, G., Tzampas, E., Protonotariou, E. and Soflanou, D. Emergence of Klebsiella pneumoniae carrying blaVIM and blaKPC genes. Hippokratia J 2010; 14(2):139-140.

13. Cagnacci, S., Gualco, L., Roveta, S., Mannelli, S., Borgianni, L., Docquier, J.D., Dodi, F., Centanaro, M., Debbia, E., Marchese, A. and Rossolini, G.M. Bloodstream infections caused by multidrug-resistant Klebsiella pneumoniae producing the carbapenemhydrolysing VIM-1 metallo- $\beta$-lactamase: first Italian outbreak. J Antimicrob Chemother 2007;61(2):296300 .

14. Zhang, F., Zhu, D., Xie, L., Guo, X., Ni, Y. and Sun, J. Molecular epidemiology of carbapenemaseproducing Escherichia coli and the prevalence of ST131 subclone H30 in Shanghai, China. European J Clin Microbiol Infect Dis 2015;34(6):1263-9.

15. Goudarzi, H., Taherpour, A., Fallah, F. and Pourkaveh, B. Laboratory detection of 
carbapenemases in gram-negative bacteria. Arch Clin Infect Dis 2016;11(2):e32816.

16. Giske, C., Gezelius, L., Samuelsen, Ǿ., Warner, M., Sundfjord, A. and Woodford, N. A sensitive and specific phenotypic assay for detection of metallo- $\beta$ lactamases and KPC in Klebsiella pneumoniae with the use of meropenem disks supplemented with aminophenylboronic acid, dipicolinic acid and cloxacillin. Clin Microbiol Infect. 2011;17(4):552-6.

17. Thomson, K.S., Extended-spectrum- $\beta$-lactamase, AmpC, and carbapenemase issues. J Clin Microbiol. 2010;48(4):1019-25.

18. Khalil, M.A.F., Elgaml, A. and El-Mowafy, M. Emergence of Multidrug-Resistant New Delhi Metallo- $\beta$-Lactamase-1-Producing Klebsiella pneumoniae in Egypt. Microbial Drug Resist 2017; 23(4):480-487.

19. Mlynarcik, P., Roderova, M. and Kolar, M. Primer Evaluation for PCR and its Application for Detection of Carbapenemases in Enterobacteriaceae. Jundishapur J Microbiol 2016;9(1):e29314.

20. Adwan, G., Bourinee, H. and Othman, S. Prevalence of Metallo- $\beta$-lactamases Producing Escherichia coli Isolated from North of Palestine. J Microbiol Antimicrob Agents 2016;2(1):9-15.

21. Amjad, A., Mirza, I.A., Abbasi, S.A., Farwa, U., Malik, N. and Zia, F. Modified Hodge test: A simple and effective test for detection of carbapenemase production. Iran J Microbiol 2011;3(4):189-193.

22. Lee, K., Chong, Y., Shin, H.B., Kim, Y.A., Yong, D. and Yum, J.H. Modified Hodge and EDTA-disk synergy tests to screen metallo- $\beta$-lactamase-producing strains of Pseudomonas and Acinetobacter species. Clin Microbiol Infect 2001;7(2):88-91.

23. Mirsalehian, A., Kalantar-Neyestanaki, D., Nourijelyani, K., Assadollahi, K., Taherikalani, M., Emaneini, M. and Jabalameli, F. Detection of AmpC- $\beta$-lactamases producing isolates among carbapenem resistant $\mathrm{P}$. aeruginosa isolated from burn patient. Iran J Microbiol 2014;6(5):306-310.

24. Goel, V., Hogade, S.A. and Karadesai, S.G. Prevalence of extended-spectrum beta-lactamases, AmpC beta-lactamase, and metallo-beta-lactamase producing Pseudomonas aeruginosa and Acinetobacter baumannii in an intensive care unit in a tertiary care hospital. J Sci Soc 2013;40(1):28-31. 25. Shahcheraghi, F., Abbasalipour, M., Feizabadi, M.M., Ebrahimipour, G.H. and Akbari, N. Isolation and genetic characterization of metallo- $\beta$-lactamase and carbapenamase producing strains of Acinobacterbaumannii from patients at Tehran hospitals. Iran J Microbiol 2011; 3(2): 68-74.

26. Damavandi, M.-S., A. Gholipour, and M.L. Pour, Prevalence of Class D Carbapenemases among Extended-Spectrum $\quad \beta$-Lactamases Producing Escherichia coli Isolates from Educational Hospitals in Shahrekord. J Clin Diagn Res 2016;10(5):DC01. 27/ Yatsuyanagi, J., Saito, S., Harata, S., Suzuki, N., Ito, Y., Amano, K.I. and Enomoto, K. Class 1 integron containing metallo-beta-lactamase gene blaVIM-2 in Pseudomonas aeruginosa clinical strains isolated in Japan. Antimicrob Agents Chemother. 2004;48(2):626-8.

28. Khorsi, K., Messai, Y., Hamidi, M., Ammari, H. and Bakour, R. High prevalence of multidrugresistance in Acinetobacter baumannii and dissemination of carbapenemase-encoding genes blaOXA-23-like, blaOXA-24-like and blaNDM-1 in Algiers hospitals. Asian Pac J Trop Med 2015; 8(6):438-46.

29. Nobari, S., Shahcheraghi, F., Rahmati Ghezelghe, F. and Valizadeh, B. Molecular characterization of carbapenem-resistant strains of Klebsiella pneumoniae isolated from Iranian patients: first 
identification of blaKPC gene in Iran. Microb Drug Resist 2014;20(4):285-93.

30. Sahin, K., Tekin, A., Ozdas, S., Akin, D., Yapislar, A., Ramazan Dilek, A. and Sonmez, E. Evaluation of carbapenem resistance using phenotypic and genotypic techniques in Enterobacteriaceae isolates. Ann Clin Microbiol 2015;14(1):44.

31. Swain, B., Otta, S. and Rout, S. Resistance pattern of Klebsiella from urinary tract infections in a tertiary care hospital. Indian Pract 2015;68(2):18-22.

32. Peleg, A.Y., Seifert, H. and Paterson, D.L. Acinetobacter baumannii: emergence of a successful pathogen. Clin Microbiol Rev 2008;21(3):538-82.

33. Lee, K., Lim, Y.S., Yong, D., Yum, J.H. and Chong, Y. Evaluation of the Hodge test and the imipenem-EDTA double-disk synergy test for differentiating metallo- $\beta$-lactamase-producing isolates of Pseudomonas spp. and Acinetobacter spp. J Clin Microbiol 2003;41(10):4623-9.

34. John, S. and Balagurunathan, R. Metallo beta lactamase producing Pseudomonas aeruginosa and Acinetobacter baumannii. Indian J Med Microbiol 2011;29(3):302-4.

35. Kumar, S. and Mehra, S.K. Performance of modified hodge test and combined disc test for detection of carbapenemases in clinical isolates of Enterobacteriaceae. Int J Curr Microbiol App Sci 2015; 4(5):255-261.
36. Kanchanadevi, P. and Sekaran, S.C. Importance of EDTA in the Detection of Metallo Beta Lactamase from Imipenem Resistant Gram Negative Bacilli. Int J Curr Microbiol App Sci 2016; 5(11):702-706.

37. Singhal, S., Mathur, T., Khan, S., Upadhyay, D.J., Chung, S.Gained, R. and Rattan, A. Evaluation of methods for AmpC beta-lactamase in gram negative clinical isolates from tertiary care hospitals. Indian $\mathbf{J}$ Med Microbiol. 2005;23(2):120-4.

38. Koshesh, M., Mansouri, S., Hashemizadeh, Z. and Kalantar-Neyestanaki, D. Identification of extendedspectrum $\beta$-lactamase genes and ampc- $\beta$-lactamase in clinical isolates of escherichia coli recovered from patients with urinary tract infections in Kerman, Iran. Arch Pediat Infect Dis. 2017;5(2):e37968.

39. Mosavian, M. and Koraei, D. Molecular Detection of IMP Carbapenemase-Producing Gram-Negative Bacteria Isolated From Clinical Specimens in Ahvaz, Iran. Jentashapir J Health Res. 2016;7(6):e36394.

40. Tawfik, A.F., Shibl, A.M., Aljohi, M.A., Altammami, M.A. and Al-Aghamy, M.H. Distribution of Ambler class A, B and D $\beta$-lactamases among Pseudomonas aeruginosa isolates. Burns J 2012; 38(6):855-860.

41. Zeighami, H., Haghi, F. and Hajiahmadi, F. Molecular characterization of integrons in clinical isolates of betalactamase-producing Escherichia coli and Klebsiella pneumoniae in Iran. J Chemother 2015;27(3):145-51. 